RUNNING HEAD: Careless Responding

Survey Mode and Data Quality: Careless Responding Across Three Modes in Cross-Cultural Contexts

Zoe Magraw-Mickelson'1, Harry Wang², and Mario Gollwitzer ${ }^{1}$

1 Social Psychology Chair, Department of Psychology, Ludwig-Maximilians-Universität München, Munich, Germany

2 Department of Management and Global Business, Rutgers Business School, Rutgers University, Newark, New Jersey, USA

Correspondence: Zoe Magraw-Mickelson magraw-mickelson@psy.lmu.de 


\begin{abstract}
Much psychological research depends on participants' diligence in filling out materials such as tests or surveys. However, not all participants are motivated to respond attentively, which leads to unintended issues with the quality of the data. Our question is: how do different modes of data collection - paper/pencil, computer/web-based, and smartphone - affect participants' diligence vs. "careless responding" tendencies and, thus, the data quality? Results from prior studies suggest that different modes of data collection produce a comparable prevalence of careless responding tendencies. However, as technology develops and data are collected with increasingly diverse populations, this question needs to be readdressed and taken further by looking at different cultural contexts. The present research examined the effect of survey mode on careless responding across three waves in a repeated-measures design. Following recommendations in the literature, we computed a careless responding index as a composite of eight indicators that capture aspects of a participant's inattentiveness. First, in a sample of working adults from China, we found that participants were significantly more careless when completing computer/web-based survey materials than in paper/pencil mode. Next, in a sample of German students, participants were significantly more careless when completing the paper/pencil mode compared to the smartphone mode. Finally, in a sample of Chinese-speaking students, we found no difference between the modes. Theoretical and practical implication were also discussed.
\end{abstract}

Keywords: careless responding, data collection, cross-cultural, data quality, survey modes Word count: 7,595 


\section{Survey Mode and Data Quality: Careless Responding Across Three Modes in}

\section{Cross-Cultural Contexts}

Psychological research often depends on self-report surveys to collect data, which depends on participants giving meaningful answers. Participants giving answers without regarding the content of the questions is a source of measurement error that can lead to unusable data or even inaccurate conclusions. Data quality remains an important, but more complex issue whilepsychological research is expanding in two significant ways: first, technological speaking, there has been a distinct shift from paper and pencil surveys to those done on computer/web platforms, to the increasing use of mobile phone platforms. With this shift, some studies suggest that these are equally effective platforms (Antoun, 2015; Casler, Bickel, \& Hackett, 2013; De Beuckelaer \& Leievens, 2009; Dodou \& de Winter, 2014). However, as humans' familiarity with and the novelty of technology is ever shifting, the quality of data generated from different platforms warrants reexamination over time. Second, while most studies that appeared in mainstream psychological journals were based on a homogeneous samples from Western, Educated, Industrialized, Rich, and Democratic (WEIRD) societies (see Henrich, Heine, and Norenzayan, 2010), an increasing number of studies now include subsamples from different countries or cultures, or try to replicate their findings in these countries/cultures in order to scrutinize their external validity (e.g., Aknin et al., 2013).

Developments in technology has led to an interest in data quality with a focus on questions such as rudimentary data screening methods, i.e. straightlining or string responses (Zhang \& Conrad, 2014) and cross-platforms comparisons (Antoun, 2015; Casler, Bickel, \& Hackett, 2013; de Bruijne \& Wijnant, 2014; Mavletova \& Couper, 2013; Yun \& Trumbo, 2000). However, there is a research gap in the cultural influences of "careless responding". With this in mind we look specifically at a sample of Chinese adults, a group otherwise not represented in the data quality and modes literature, to answer the question: how do different 
modes of data collection - paper/pencil, computer/web-based, and smartphone - affect participants' diligence vs. "careless responding" tendencies and, thus, the data quality?

Early research in the comparison of survey modes has largely focused on a comparison of completion rates (de Leeuw \& de Heer, 2002; Dillman, Smyth, \& Christian, 2014; Groves, 2006, Tortora, 2009). This is an important metric for market research and data panels because non-response can introduce systematic bias (Brosnan, Grün, \& Dolnicar, 2017). However, results are ambiguous with regards to differences between completion rates for different modes. Research comparing paper/pencil to computer/web-based surveys show higher response rates with paper/pencil surveys (Hoonakker \& Carayon, 2009; Sax, Gilmartin, \& Bryant 2003). While the majority of research shows that smartphone surveys have worse completion rates compared to web surveys (e.g. de Bruijne \& Wijnant 2014; Mavletova and Couper, 2013) and some studies find no difference (e.g., Antoun, 2015). Furthermore, survey completion does not necessarily equate to data quality. In data cleaning, it is common practice to exclude incomplete data points or implement survey design that requires a response to all questions (possible with online platforms). Therefore, the question remains what are the differences in modes for data that is completed in regards to quality, specifically as defined by careless responding.

Careless responding (CR) is defined as data that “doesn't reflect respondents" true levels of the construct purportedly being measured" (Meade \& Craig, 2012). In the literature, this has also been referred to as insufficient effort responding (Bowling et al., 2016; Curran, 2016, 2016; Huang Curran, Keeney, Poposki, \& DeShon, 2012), inattentive responding (Maniaci \& Rogge, 2014), and satisficing (Fang, Wen, \& Prybutok, 2014), all of which describe a response style that is random in regard to the item's content. This is conceptually different from response bias, which is a nonrandom source of error. Although random (i.e., unsystematic) measurement error primarily affects the reliability of a measure, it can, under certain circumstances (e.g., large deviations from the mean), also increase the likelihood of 
systematically biased results (e.g., inflating alphas; cf. Barnette, 1999 and regression to the mean; cf. Huang, Liu, \& Bowling, 2014; Goldammer et al., in press; see also Gollwitzer, Christ, \& Lemmer, 2014).

Furthermore, past research has highlighted areas in which CR is problematic for psychometric outcomes. For example, in scale development, Wood (2006) showed that the expected one-factor solution was rejected if ten percent or more of the items were completed carelessly. While Oppenheimer et al. (2009) found greater statistical power in samples that excluded participants with identifiable careless responding behavior. Additional research calls similar attention to the adverse effects of CR on correlations and internal consistency (Meade \& Craig, 2012; Ward, Meade, Allred, Pappalardo, \& Stoughton, 2017).

In contrast to these sample-centric research on careless responding (CR), another line of research focuses on data screening, such as looking at response time and eliminating cases with impossibly fast times can catch some of the most extreme cases of careless responding; however, these methods can be fallible because they tend to overlook cases in which responses were careless, albeit within plausible response time ranges. Suggested cut-off for response time are inherently conservative (Curran, 2016), while removing CR respondents can lead to systematic bias that can limit the generalizability of findings (Meade \& Craig, 2012). Therefore, the approach we take from the literature is to look at an array of careless responding $(\mathrm{CR})$ indices instead of a single indicator when contrasting different modes of survey responses in a cross-cultural context

This is advantageous for many reasons. First, any single method would miss other types of CR, there are many ways in which a participant's response might not reflect the questions at hand. Second, many methods are designed to catch responders who are equally careless throughout the whole survey, in response to each and every question, which is often not typical careless behavior. For example, a common method is to use single item questions, such as "use me" prompts (e.g., "In your honest opinion, should we use your data in our 
analyses in this study?") or bogus questions (e.g., "I sleep less than one hour per night.") at one point in the survey. Such approaches are likely to produce a type-1 error (i.e., falsely flagging a response as "careless") or a type-2 error (i.e., failing to flag CR as such). Finally, this approach has been used widely in the careless responding literature "to avoid potential idiosyncratic effects attributable to any single IER [CR] index" (Huang, Liu, \&Bowling, 2014, also see Bowling, et al., 2016; Grau, Ebbeler, \& Banse 2019).

An extensive review of the literature came up with twelve common measures that are used to calculate a careless responding index: response time, long string max, long string mean, Mahalanobis distance, person total correlation, even-odd consistency, psychometric synonyms, psychometric antonyms, self-reported effort, a self-reported single 'use me' item, bogus questions and instructed response items (Bowling et al., 2016; Curran, 2016;

DeSimone, Harms, \& DeSimone, 2014; Grau et al., 2019; Huang et al., 2012; Huang, et al., 2014; Huang, Bowling, Liu, \& Li, 2015; Johnson, 2005; Maniaci \& Rogge, 2014; Meade \& Craig, 2012; Goldammer et al., in press; Ward et al., 2017). We will now take a closer look at these measures and what types of careless responding tendencies they capture.

Response time is one of the most common data screening methods. It is normally calculated by determining the minimum time required to complete a survey or a block of questions. A cutoff value is then used to exclude participants who complete the survey in an impossibly or implausibly short amount of time. This identifies careless responders whose motive is to go through survey materials as quickly as possible.

Long-string analysis: Long-string max or mean is the analysis of string responses, measuring the number of times a respondent answered with an unbroken sequence on a Likert scale. As an index, this can be calculated as the most identical responses in a row across measures, across pages of a survey, or as the mean of the longest responses across pages. This method identifies careless responders who put little or no effort into varying their responses. Slight deviations from a single response value may not be identified by this measure. 
Mahalanobis distance is used to identify outliers that arise due to careless responding (Bowling et al., 2016; Meade \& Craig, 2012; Ward et al., 2017). By computing the participants' distance from the average participants' response pattern, this method identifies unusual data points relative to other data points in the sample using a multivariate technique. Individual consistency: this set of indices can be grouped under the assumption that attentive participants will respond in an internally consistent way. There are different methods to determine individual consistency. One such method is, psychometric synonyms, items that have a high inter-person-correlation in the overall data set that are then correlated at the individual level, the logic being that participants who have low or negative correlations were not attentive to the content of alike questions. Similarly, psychometric antonyms find the intra-person correlation for the items that are found to be most negatively correlated across the data set. This could, for example, identify participants who ignored reverse coded items. Person-total-correlation is another measure of individual consistency that measures each person's consistency of responding with that of all other participants. A negative correlation for this measure identifies individuals who responded in a contrasting way to the pattern set out by all other participants. Even-odd consistency splits unidimensional scales into two subscales, even numbered and odd numbered items, and finds the correlation between the average of these two halves, measuring the consistency in answering items of the same scale.

Self-report items: Self-reported effort asks participants to disclose how much effort they put into the task with questions such as "I worked to the best of my abilities in this study" (Meade \& Craig, 2012). A self-reported 'use me' item is usually a single item similar to self-reported effort in which the participant tells you if they think they were sufficiently attentive. These measures depend on the honesty of the participant and their attentiveness when the question is asked. However, these measures capture the fact that the participant is the one who knows best if they were careless in their responding.

Instructed response items and bogus questions are similar in that they have a right or 
wrong answer and depend on participants reading the text of the question to get the correct answer. Instructed response questions tell the participant which response to select (e.g., "Please select answer choice 5 for this question") while bogus questions present a statement with only one plausible answer.

The literature gives no definite answer on how to classify careless responding. Some argue that $\mathrm{CR}$ is an aspect of personality, with a considerable degree of consistency across time and situations, and meaningful associations with other personality dispositions, such as agreeableness (Bowling et al., 2016; Ward et al., 2017). Additionally, it has sometimes been conceptualized as an individual and country level variable that correlates with other country level variables such as cultural emancipation (Grau et al., 2019). However, there are also significant environmental factors that influence the amount of attention in the moment, such as survey mode (i.e., paper/pencil, computer-based).

Past research in CR has identified a number of design features that can be used in surveys to minimize participants CR behavior. For example, survey instruction design, and inperson and virtual participant oversight (Francavilla, Meade, \& Young, 2019; Ward \& Meade, 2018; Ward \& Pond III, 2015). However, in many research projects, interventions like increased experimenter supervision while participants complete the survey, are not typically feasible to implement. Furthermore, although these features are important, the literature does not yet have a definitive answer to the more basic question regarding difference between survey modes. This study aims to look at the role of mode above other design features.

In a within-participant design, we will look at careless responding across three modes: paper/pencil, computer/web-based, and smartphone-based. This design is particularly beneficial because we can directly compare how participants react to different modes beyond compounding individual differences, as past research has shown a connection between CR and personality variables (Bowling et al., 2016; Ward et al., 2017). Additionally, the surveys are presented as typical research is conducted, giving the participant wide latitude in the 
specifics of the environment where the survey was completed, which increases the generalizability of the results.

In formulating our hypothesis with regards to differences in CR across survey modes, one plausible assumption is that CR is more likely to occur in situations in which participants feel that they cannot be held accountable for their responses. Research shows that in-person monitoring of internet-based surveys can decrease CR for some indicators (Francavilla et al., 2019). Thus, if accountability is a relevant factor, CR should be less likely to occur in face-toface interviews (where accountability is highest) compared to paper/pencil questionnaires that are distributed and collected by a researcher or other authority (where accountability is comparably weaker), and most likely to occur in online surveys that are completed in an entirely private environment (i.e., at home; Antoun, 2015; Ward et al., 2017).

While this hypothesis makes a lot of intuitive sense, the research findings on survey mode are mixed. For instance, Johnson (2005) compared personality inventory data collected through a web-based platform to an independently collected paper/pencil data and found that data collected online were of lower quality concerning the long string indices and the number of missing responses. However, there were no differences in the individual constancy measures (Johnson, 2005). Further, we can look at mode differences in social desirability, which is relevant because social desirability can be seen as a proxy for accountability (cf. Paulhus, 1984) and is therefore related to diligence in careless responding. Dodou and de Winter (2014) looked at the effect of survey mode: paper/pencil compared to computer-based, on social desirability indicators - in a meta-analysis with 51 primary studies comprising, in total, more than 16,000 participants. Interestingly, these authors found no relation between survey mode and social desirability. Following this, in comparing smartphone to web/computer-based modes with a within-subject design, Mavketiva and Couper (2013) found mixed results - in two of the five indices they used in order to measure social desirability there was a significant different between modes. Specifically, there was more 
socially desirable responding in the smartphone responses, while, there were no significant difference in the other three indices.

However, it should be noted that the vast majority of primary studies included in Dodou and de Winter's (2014) meta-analysis as well as other CR research are conducted with Western participants. With survey diligence, accountability and social desirability concerns, there are reasons to believe that culture plays a significant role. More precisely, cross-cultural research suggests that "saving face" is an important concern in Asian cultures (Oetzel et al., 2001). Thus, one could argue that Asian participants might feel more accountable for their responses when there is face to be saved, that is, in face-to-face interactions. That would mean more careful responding when completing a paper/pencil-based questionnaire compared to a more anonymous online-based survey because with a paper/pencil-based questionnaire the participant has face-to-face contact with the experimenter. Some preliminary evidence for this is seen in Lee et al. (2018), which compared surveys modes computer/web, smartphone and phone interview in a sample of South Korean students. In addition to finding greatly increased completion rates with the phone interview mode, they also found consistently higher socially desirable responding in the responses to sensitive questions in the phone interview mode compared to the webs modes (Lee et al., 2018). Consequently, a higher degree of CR in computer/web surveys compared to paper/pencil questionnaires may be observable in Asian, but not necessarily in Western cultures. This hypothesis will be explored in the present study. Prior methodological research on survey mode and data quality has focused on the difference between paper/pencil questionnaires versus computer/web surveys, where "computer" usually meant a stationary machine located in a specific, familiar environment (e.g., one's study). However, with the increasing popularity of smartphones, many people do not even own a stationary machine anymore; more and more people do their online businesses exclusively on their smartphone. Consequently, more and more online surveys are now completed and even specifically designed for being completed on smartphones (Peterson, 
Griffin, LaFrance, \& Li, 2017). This raises the question of whether careless responding is more or equally likely to occur when the online-survey is completed on a smartphone compared to a stationary computer. One plausible assumption could be that situational circumstances are much less standardized and much "noisier" (also in a literal sense) when the survey is completed on a smartphone compared to a stationary computer. Hence, careless responding should be more likely to occur when data are collected via smartphone than via computer or paper/pencil. This hypothesis will also be explored in the present study.

Past work that looked at differences in smartphone responses confirms through selfreport measures that smartphone survey respondent report more multitasking while completing the survey on a mobile device compared to a computer (Antoun, 2015; Mavletova \& Couper, 2013). Taking a closer look at the effect of multitasking on data quality, Antoun (2015) compared sufficing behaviors, which can be another term for CR, between smartphone and computer modes using a within participant design and found no interaction. However, the concept of sufficing behaviors defined as "providing adequate but not optimal responses", as conceptualized by Antoun (2015) does not adequately fit the careless responding definition or literature we apply here. In an extension of this analysis, Antoun, Conrad, Couper and West (2019) investigated the difference in measurement errors between the two modes as conceptualized as the difference between participants responses for specified question at the two time points. In their analysis, they positioned the web/computer version as the benchmark assuming it was the more accurate of the two. In this design, they found no evidence for survey mode bias alone but rather bias due to phone ownership (That is differentiating between participants who owned a smartphone and those who completed the survey on borrowed smartphones; Antoun et al., 2019). The current study will build on this work by investigating this hypothesis with regards to data quality as measured by $\mathrm{CR}$, as well as looking at the comparison of smartphone surveys to computer/web and paper/pencil surveys.

Furthermore, the present study goes beyond past work by being one of the first to look 
at careless responding in a sample of Chinese working adults. We choose this sample firstly because there is limited research on CR in Asian samples. We aim to expand beyond the disproportionately high percentage of research that uses Western samples, which tend to use American university students or Western based survey panels (see Mavletova \& Couper, 2013 for an exception where data was collected from a Russian data panel). Second, collecting data from Chinese working adults allows for the opportunity to test our culture specific hypothesis relating to how "face" may influence CR behavior.

The specific items and scales used in the survey were selected on the basis of the following rationale: first, it was important to have a typical psychological survey that a researcher would use. Second, we included a dimension related to survey engagement. Third, we included personality factors hypothesized to be related to survey engagement. Finally, we wanted to keep the overall length of the survey short so as not to create an undue burden on our within-participant design. The format of the survey was Likert type scales optimized for mobile platform as research has shown non-optimization can affect usability (de Bruijne \& Wijnant, 2014) and to avoid special response formats that may influence quality in web versus smartphone modes (Antoun, 2015; Wells, Bell \& Link, 2014).

A careless responding (CR) index was calculated for each participant and each survey mode on the basis of participants' responses to these scales and items. CR values were then compared between survey modes (i.e., paper/pencil, computer/web, smartphone). The following hypotheses were tested:

H1: The degree of careless responding in the computer/web mode will be higher than in the paper/pencil mode.

H2: The degree of careless responding will be higher in the smartphone mode compared to the paper/pencil and computer/web mode.

Furthermore, we included the exploratory factor gender to look at how gender may affect careless responding. Although past research does not point to any gender differences it 
may nonetheless play a role, as careless responding was shown to be related to some personality traits and personality trait can be related to gender. For example, women are more agreeable than men (Lehmann, Denissen, Allemand, \& Penke, 2013) and low agreeableness has been shown to be related to more careless responding (Bowling et al., 2016; Ward et al., 2017).

\section{Study 1: Chinese adults}

\section{Methods and Materials}

\section{Participants}

Participants were asked to complete one survey in a directed mode: Paper/pencil, online with a computer, and with a smartphone, each about two weeks apart. To replicate normal survey conditions, there was no time limit for how long participants could take on any of the survey modes. The order of modes in which the surveys were completed were systematically varied (so that each mode occurred once at each order position; i.e., a Latinsquare design to minimize artificial order effects). For the paper/pencil version, the survey was given to participants on the scheduled day and requested to be returned within a week. For the computer and smartphone versions, instructions were emailed to participants and a reminder sent if not completed.

The sample of Chinese working adults $(\mathrm{N}=78)$ were all employees at a municipal clerk's office in China . The procedure was carried out by the coordination office, and they received no reward for their participation. Participants were explicitly told that personal information, which included email, ID numbers and phone numbers, would only be used for matching between the survey modes. As soon as the three modes were matched to one participant, an anonymous code would be used and their personal information would be deleted. Furthermore, we assured participants that their responses would not be seen by their supervisors.

Excluded from the analysis were entries that did not follow directions for the 
completion mode (completing the smartphone version on the computer/web), or that entered more than one response for a particular survey mode (Chinese working adults: $\mathrm{N}=4$ ). For these participants, only the dataset that was completed first was retained. Demographics are presented in Table 1.

\section{Materials}

The base of the survey included demographics (age, gender, education level, tenure/semester in university) as well as the following measures, all completed on six-point Likert scales ranging from 0 (“do not agree at all”) to 5 (“agree completely”): Agreeableness from the Mini- International Personality Item Pool (IPIP) (Donnellan et al., 2006), which consists of four items (e.g. "I sympathize with others' feelings"); social desirability, 6 items (e.g. "I sometimes tell lies") from the Balanced inventory of desirable responding (BIDR) Version 6 short form (Stöber et al., 2002; BIDR used in China: Fang et al., 2014); a short harmony motivation scale (adapted from Leung, 2010,) which includes harmony enhancement, 5 items, (e.g. "Being patient and willing to compromise is a show of respect to the other person.") and disintegration avoidance, 5 items, (e.g. "You should not create conflict. When you have a conflict, you should try to smooth it over and make the other person happy."); the four subscales from the Justice Sensitivity Inventory (Schmitt et al., 2010, i.e., Victim Sensitivity, Observer Sensitivity, Beneficiary Sensitivity, and Perpetrator Sensitivity, 10 items each); justice heuristics (Ambrose \& Schminke, 2009), 6 items (e.g. "In general, I can count on my supervisor/instructors to be fair.”); attitudes towards surveys (Stocké, 2006, adapted), 3 items (e.g., "Participation in surveys is generally useful.”); technology ease of use (adapted from Saadé \& Bahli, 2005,), 4 items (e.g. "Using [this questionnaire / this computer / this smartphone app] for this survey is easy for me to do.”); feelings of anonymity, 3 items (e.g. "I felt anonymous completing this survey"); one item for work stress (e.g. "Overall, the stress I feel at work is acceptable”), and self-reported effort, 4 items (e.g. "I worked to the best of my abilities in this study;", Meade \& Craig, 2012) . 


\section{Computing a Careless Responding Index}

Because this design included a paper/pencil survey, response time could not be calculated. Even-odd consistency was also not a good fit as an index for this data set because it requires a comparison between the odd and even questions across various sufficiently long univariate scales. This is known to be a limitation of this technique (Curran, 2016). Furthermore, we only included three instructed response items, as recommended by Meade and Craig (2012). These are preferable to bogus questions and because of the short length of our survey, we did not want to annoy participants with too many of this type of question. Although we collected a Yes/No 'use me' question, we did not include this in the index because of its dichotomous nature. Finally, we opted to use long string mean as the longstring analysis measure.

Therefore, we included seven indicators of careless responding in the composite index. In the following, we will describe the method used to calculate each of the indices.

To calculate long string mean, we used three pages or blocks of questions that included different measures which appeared on the same page (Block 1: agreeableness and social desirability; Block 2: attitudes, ease of use, anonymous, effort; Block 3: harmony enhancement). Long string mean was the average of long string answers across three blocks.

Mahalanobis distances: to calculate this measure, we averaged the Mahalanobis distances of nine scales: Justice sensitivity scales (3), Agreeableness, Social Desirability, Harmony Enhancement, Disintegration Avoidance, and Justice Heuristics. As seen in Table 2, for our data sets, the correlation with Mahalanobis distances and other indices is rather low compared to those reported in past studies that report similar measures. This may be due to the distribution of the data, which is skewed rather than evenly distributed. Past research has shown that Mahalanobis distances' utility as an indicator is sensitive to the data's distribution (Meade \& Craig, 2012).

Psychometric synonyms: Curran (2016) recommended identifying all inter-correlated 
pairs without repeat items above the cut-off of $r=.60$ between items. However, that strategy depends a great deal on the nature of the sample. Therefore, to establish some consistency across our three samples, we took the ten highest positively correlated item pairs, rs $>.61$. For psychometric antonyms, there are also recommendations for negative correlation cut-offs values of $r=-.20$. However, we again decided to use a set number of pairs for each sample. Here, we used five pairs because the survey was not very long and did not include a large number of reverse coded items rs $<-.30$. Once the pairs of items were identified, the intraperson correlation of those item sets was computed for each participant. When the correlation of synonyms or antonyms was not calculable due to the lack of variance, meaning either in the numerator or dominator, the participant had the same value for all items in the selected set, and the indices for that participant were entered as a missing value.

Person-total-correlation scores were calculated across 60 items (i.e., justice sensitivity scales, agreeableness scale, social desirability scale, harmony enhancement, and disintegration avoidance scales) exactly as described by Curran (2016).

For self-reported effort, we used a four-item measure as described above. For a full list of questions, see Appendix A.

For instructed response items, we included three questions per wave. An example of these questions is: "Please check 'very accurate' to show you are paying attention". Answers that matched "very accurate" were coded as 0 , and all other responses were coded as 1 . The values were summed across three questions; therefore, the possible range of this measure was $0-3$.

For the self-reported 'use me' item, we used the single item from (Meade \& Craig, 2012): item "In your honest opinion, should we use your data in our analyses in this study?" Yes was coded as 0 , and No coded as 1 .

To compute the CR index for each person, we multiplied person-total-correlation, psychometric synonyms, and self-reported effort by -1, so that all measures of CR are scaled 
in the same direction (i.e., higher values indicating a stronger carelessness). Next, we ztransformed (i.e., standardized) the seven indices and then computed an aggregate score by averaging across indices. See Table 2 for the correlations between the indices.

\section{Results}

We ran one-factorial mixed models. Descriptive statistics are reported in Table 3.

We looked at the CR index to investigate the contrast of our main hypothesis. We included two Helmert-coded contrasts: (1) paper/pencil $=-1$, smartphone $=0$, and computer/web $=1 ;(2)$ paper/pencil $=-1$, smartphone $=2$, and computer/web $=-1$. Thus, the first contrast compares paper/pencil against computer/web (as specified in Hypothesis 1), and the second contrast compares the smartphone mode to the two other modes (as specified in Hypothesis 2). Given that both Hypotheses were directional, the contrasts were tested for statistical significance on a 5\% level (one-tailed).

$\mathrm{H} 1$ : We found evidence for $\mathrm{H} 1$, responses were more careless in the computer/web mode compared to paper/pencil mode: $\mathrm{B}=.06, \mathrm{SE}(\mathrm{B})=.03, \mathrm{p}=.04$ (see Figure 1).

$\mathrm{H} 2$ : We did not find evidence for $\mathrm{H} 2$, responses were not more careless in the smartphone mode compared to the other two modes, $\mathrm{B}=-.02, \mathrm{SE}(\mathrm{B})=.02, \mathrm{p}=.23$.

\subsection{Individual indices}

Looking at the different indices individually, in line with $\mathrm{H} 1$ we found that, for psychometric antonyms and person-total-correlation, participants showed significantly more carelessness in the computer/web mode compared to the paper/pencil mode $(\mathrm{B}=.14$, $\mathrm{SE}(\mathrm{B})=.07, \mathrm{p}=.02$, and $\mathrm{B}=.13, \mathrm{SE}(\mathrm{B})=.06, \mathrm{p}=.02$, respectively).

\section{Additional exploratory findings}

In an exploratory fashion, we investigated gender differences in careless responding we found that men were significantly more careless than women in the paper/pencil mode condition, $\mathrm{B}=-.36, \mathrm{SE}(\mathrm{B})=.09, \mathrm{p}<.001$, in the computer/web mode condition, $\mathrm{B}=-.47$, $\mathrm{SE}(\mathrm{B})=.12, \mathrm{p}<.001$, but not in the smartphone condition, $\mathrm{B}=-.18, \mathrm{SE}(\mathrm{B})=.11, \mathrm{p}=.11$. 


\section{Discussion}

The results of this first study found evidence for Hypothesis 1: in an East Asian culture that puts emphasis on face, in the mode that required more interaction with the coordinator, that is, paper/pencil, participants were less careless compared to computer/web mode. However, we did not find evidence for Hypothesis 2 (evidence of more careless responding in the smartphone mode).

In order to confirm a culture specific nature of Hypothesis 1 and to replicate our findings for Hypothesis 2, we decided to conduct the same experiment in Germany. Without the same access to an equivalent work force, Study 2 was conducted with students at a German university.

\section{Study 2 German students}

Our second study with a German student sample was conducted to replicate study one and to investigate the culture specific nature of Hypothesis 1, which states that the result should not extend to a Western sample. However, we maintain our Hypothesis 2 as previously stated.

In line with past research in a Western context, we do not predict a difference between the computer and paper/pencil modes:

H1: There will be no significant difference in the degree of careless responding for the computer/web mode compared to the paper/pencil mode.

Although the Chinese adult sample did not show more carelessness in the smart phone version, given the lack of relevant past research and the possibility of cultural difference, we maintain the following:

$\mathrm{H} 2$ : The degree of careless responding will be higher in the smartphone mode compared to the paper/pencil and computer/web mode.

\section{Participants}

The German students in the sample $(\mathrm{N}=117)$ were primarily first-year psychology 
students . The procedure was administered through a mandatory course, and the paper/pencil version of the materials was followed by other unrelated survey measures for which the students received course credits for completing all parts. In all conditions, participants were told the survey was for university research and that their participation was completely voluntary.

Excluded from the analysis were entries that did not follow directions regarding completion mode (completing the smartphone version on the computer/web or vice versa), and therefore entered more than one response for a particular version $(\mathrm{N}=32)$. For these participants, only the dataset that was completed first was retained. Included in the calculation and examination of CR but excluded from the analysis of differences between modes were participants who completed only one version $(\mathrm{N}=51)$. Demographics are presented in Table 1 .

\section{Materials and Methods}

The material and method for computing CR were the same as in Study 1.

\section{Results}

H1: We found no evidence of difference in the degree of careless responding in the computer/web mode compared to the paper/pencil mode, $\mathrm{B}=-.04, \mathrm{SE}(\mathrm{B})=.03, \mathrm{p}=.07$ (see Figure 2).

H2: In the German student sample, we also did not find evidence for $\mathrm{H} 2$; in fact, we found marginally significant results in the opposite direction: responses were even more careless when made on the computer/web and the paper/pencil mode compared to the smartphone, $\mathrm{B}=-.02, \mathrm{SE}(\mathrm{B})=.01, \mathrm{p}=.07$.

\section{Individual Indices}

In self-reported effort, contrary to Hypothesis 1 , German students were significantly more careless in the paper/pencil mode compared to computer/web, $\mathrm{B}=-.13, \mathrm{SE}(\mathrm{B})=.07$, $\mathrm{p}=.03$. Counter to Hypothesis 2 , for the measure of psychometric synonyms, participants showed significantly more carelessness in the paper/pencil and computer/web mode compared 
to the smartphone mode, $\mathrm{B}=-.09, \mathrm{SE}(\mathrm{B})=.05, \mathrm{p}=.04$. The instructed response items and 'use me' item significance was not calculated because there were so few in the German sample who missed these questions, and in some modes none at all.

\section{Additional exploratory findings}

In our exploratory analysis, we found that men were significantly more careless than women in the computer/web mode $(\mathrm{B}=-.44, \mathrm{SE}(\mathrm{B})=.16, \mathrm{p}=.01)$, but not in the other two modes, $\mathrm{p}>.06$.

Finally, to follow up on the marginally significant results of Hypothesis 2, we tested the difference between smartphone and computer/web as well as the difference between smartphone and paper/pencil separately. We found a significant difference in paper/pencil compared to smartphone, $\mathrm{B}=-.03, \mathrm{SE}(\mathrm{B})=.02, \mathrm{p}=.02$, but not computer/web compared to smart phone, $\mathrm{B}=-.03, \mathrm{SE}(\mathrm{B})=.02, \mathrm{p}=.15$.

\section{Discussion}

The results from Study 2 were as predicted concerning Hypothesis 1 and unexpected with regards to Hypothesis 2 . Although we possibly confirmed the culturally specific nature of Hypothesis 1, there is still the open question about whether these differences are due to culture or age as samples in Study 1 and 2 differed in both.

Therefore, in Study 3, we sampled Chinese students, who were more comparable to the sample used in Study 2. With the rapid rise of technology as described above, there are noticeable generational differences in how people interact with technology. Prensky (2001) describes the difference between Digital Natives, those who grew up with computers, video games and the Internet, and Digital Immigrants who came to these technologies later in life and how this leads to profound difference in how these groups interact with technology as well as in how they think and process information. More recent research supports this and shows generational difference in areas such as engagement with social media (Bowe, \& Wohn, 2015), adoption and use of information and communications technology (Lee, \& 
Coughlin, 2015), and frequency of use (Olson, O'Brien, Rogers, \& Charness, 2011). Thus, it is important to look at both age groups and culture when investigating participants' interaction with technology. Study 3 achieved this by looking at a Chinese student sample.

\section{Study 3 Chinese students}

In Study 3, our aim was to clarify the difference found in the previous two studies concerning age and culture. That is, were the discrepancies in the results found with the previous two samples due to the difference in culture or the difference in age cohort? Although it is possible that both these factors influence participants' careless responding, our hypotheses are the same for the Chinese students as for the Chinese adults:

H1: The degree of careless responding in the computer/web mode is higher than in the paper/pencil mode.

$\mathrm{H} 2$ : The degree of careless responding will be higher in the smartphone mode compared to the paper/pencil and computer/web mode.

\section{Participants}

The Chinese speaking student sample came from two locations: first, from University $1(\mathrm{~N}=74)$, wherein the procedure was administered through university language courses and second, from University $2(\mathrm{~N}=39)$, where the surveys were administered by a teacher in their organizational psychology course . Both Chinese-speaking student locations received no reward for participating. Again, participants were told the survey was for university research and that their participation was completely voluntary.

Excluded from the analysis were entries that did not follow directions regarding completion mode (completing the smartphone version on the computer/web or vice versa), and therefore entered more than one response for a particular version $(\mathrm{N}=22)$. For these participants, only the dataset that was completed first was retained. Participants who completed only one version $(\mathrm{N}=42)$ were included in the calculation and examination of $\mathrm{CR}$ but excluded from the analysis of differences between modes. Demographics are presented in 
Table 1.

\section{Materials and Methods}

The material and method for computing CR were the same as in the previous studies.

\section{Results}

$\mathrm{H} 1$ : In the Chinese student sample, we did not find evidence for $\mathrm{H} 1$; responses were not more careless in the computer/web mode compared to paper/pencil mode, $\mathrm{B}=.02$, $\mathrm{SE}(\mathrm{B})=.04, \mathrm{p}=.27$ (see Figure 3).

$\mathrm{H} 2$ : In the Chinese student sample, we did not find evidence for $\mathrm{H} 2$; responses were not more careless in the smartphone mode compared to the computer/web mode and the paper/pencil mode, $\mathrm{B}=.00, \mathrm{SE}(\mathrm{B})=.02, \mathrm{p}=.43$.

\subsection{Individual indices}

There were no significant differences between the modes and any of the indices for the Chinese student sample.

\section{Additional exploratory findings}

With regards to gender differences between modes we did not find any significant differences ps $>.09$.

\section{Discussion}

The results of Study 3 were unexpected in that the participants did not differ in their careless responding between modes. With this sample of Chinese students we found evidence neither for Hypothesis 1 (that the Chinese samples would be more careless in the computer/web mode than in the paper/pencil mode), nor for Hypothesis 2 (that participants would be more careless on the smart phone than the paper/pencil or computer/web mode).

\section{General Discussion}

Past research has shown that careless responding is inconsistently different between self-report assessment modes. We challenged these results by looking more closely at careless responding tendencies in three different survey modes (paper/pencil, computer/web, and 
smartphone) and in three different samples (Chinese working adults, German students, Chinese students). Looking at each of these three samples in succession, and using an aggregated careless responding score consisting of seven different indicators, we found that (1) Chinese working adults were more careless when responding on a computer compared to when responding with paper and pencil; (2) German students were more careless responding with paper and pencil compared to on a smartphone; and that (3) there were no significant differences in careless responding between modes in the Chinese students sample. Thus, only one of our hypotheses (H1) was corroborated by the data and in only one sample, while Hypothesis 2 could not be confirmed. Contrary to the assumptions of past work in careless responding (Ward et al., 2017), we did not find evidence that smartphone surveys were completed with more carelessness than surveys completed on other platforms (H2); in fact, we found the opposite with the German student sample. In the following section, we will discuss our findings against the background of other research that has been conducted in this area.

\section{Careless Responding in Chinese Samples}

Fang, Wen, and Prybutok (2014) found that university students in China were more likely to engage in sufficing behavior-defined as a suboptimal response style characterized as reduced cognitive effort when responding to a survey — on a computer compared to a paper and pencil survey. These results are consistent with our Chinese working adults sample but not the Chinese student sample. This points to generational and cultural differences in familiarity with different survey modes. The student samples used in Fang, Wen, and Prybutok (2014) are represented in the present study as the working adults' sample; this cohort might have a different relationship to with technology compared to the current student sample. This is plausible given that age cohorts are growing up with different set of technological norms than just a few years ago.

\section{Careless Responding in the German Students Sample}


The results of the German sample were unexpected. Previous research has not found a similar pattern; however, we can speculate on the relationship between environment and careless responding. Our hypothesis relied on the assumption that participants would be more careless in a more distractible environment. However, this might not have been the case. The German students received the paper and pencil survey in class, even though it was not related to their class work and they may have completed it while still amongst their peers engaged in discussion. On the other hand, on the smartphone participants may be more singularly focused compared to the paper/pencil and computer/web mode and therefore less careless (e.g. imagine the comparison of a classroom to students sitting on a bus and using a smartphone, not interacting with other passengers around them). Future research should collect more information regarding the ability to focus in different environments where surveys are completed to better understand the differences between survey modes. Finally, one further differentiation is that the paper/pencil mode was followed by other unrelated survey measures, we cannot rule out the effect of this on the students' behavior. However, we think it is unlikely as an explanation for our results because the relevant portion of the survey was at the very beginning and in a distinct format (that matched the other survey modes, but not the rest of the irrelevant sections).

\section{A More Fine-Grained Analysis of Careless Responding}

Looking at single indicators of careless responding, we found similar patterns for the difference between the modes for each country; however, these differences only reached significance for person-total-correlation and psychometric antonyms in the Chinese working adults sample and psychometric synonyms and self-reported effort in the German student sample. This further supports the concept of a composite measure to capture different types of CR because looking at only one or a small number of indicators would not have shown the complete picture in the differences between modes in carless responding.

We also found exploratory results related to gender. Although gender itself is a 
meaningless psychological variable and therefore unable to "explain" our pattern of results, it is important to note we found that men were more careless than women in two modes in the Chinese adult sample and in the computer mode for the German student sample. This could be related to the gender difference in agreeableness (Lehmann, Denissen, Allemand, \& Penke, 2013) and the fact that agreeableness has been shown to be related to careless responding (Bowling et al., 2016, Ward et al., 2017). However, it is unclear why this difference would only be seen in the computer mode for two of the samples. Future research should explore this issue.

\section{Limitations}

The limitations of this research design is that although we can describe differences between survey modes and cultures, it is hard to look at the psychological mechanisms behind survey differences, using survey questions that depend on the participant's response. We asked questions related to possible sources of differences in careless responding, such as ease of use and anonymity. However, if careless responders are not paying attention to the content of these questions, we will not have a reliable comparison. Future research should look into novel ways to combat this issue.

In computing the careless responding index, we took the average of the centered values across seven indices. This is a strength, in that we took into account various types of careless responding, but it is also a limitation in that it assumes all the indices are equally good at identifying careless responders.

Cut-offs for exclusion criteria based on careless responding can be difficult to prescribe and are beyond the scope of this article. Some of the indices have clear dichotomous cut-offs; however, these do not catch all different types of careless responders. Composite indices can be very useful for that; however, more research needs to be done to provide recommendations that researchers can use to identify careless responders. This can be done through simulation studies, which can control the percentage of careless responding and vary 
the patterns of randomness to see how a CR index performs, along with experimental studies that look at careless responding with novel and labor-intensive measures that can be compared to a universally applicable index as the one used in this paper.

Furthermore, this study compared modes across a relatively short psychological study. Future research should look at studies of different length and different types of measures. In addition, we did not collect information on the type of devices used. With smartphones becoming increasingly larger and some computers having touch screen abilities, the distinction between these modes may blur, and future research should take this into account (e.g. Brosnan, Grün, \& Dolnicar 2017; Tourangeau, et al., 2017; Wells, Bailey, \& Link, 2013).

\section{Conclusion}

This research looked at careless responding in a typical research environment. Our results suggest that data quality is not equal across modes of data collection and it is not the same across different cultures and groups: computer/web is best avoided when surveying Chinese working adults; smartphone is preferred when surveying German speaking students, and Chinese speaking students are indifferent to different survey modes. Clearly, more work needs to be done, but the present study is a first, and, arguably, important step towards understanding situational factors, cultural factors, and gender in terms of how careful participants are when completing surveys. 


\section{Conflict of Interest}

The authors declare that the research was conducted in the absence of any commercial or financial relationships that could be construed as a potential conflict of interest. 


\section{REFERENCES}

Aknin, L. B., Barrington-Leigh, C. P., Dunn, E. W., Helliwell, J. F., Burns, J., Biswas-Diener, R., ... \& Norton, M. I. (2013). Prosocial spending and well-being: Cross-cultural evidence for a psychological universal. Journal of Personality and Social Psychology, $104,635-652$.

Ambrose, M. L., \& Schminke, M. (2009). The role of overall justice judgments in organizational justice research: a test of mediation. Journal of Applied Psychology, 94, 491-500.

Antoun, C. (2015). Mobile Web Surveys: a First Look at Measurement, Nonresponse, and Coverage Errors. (Doctoral dissertation). University of Michigan. Retrieved from http://deepblue.lib.umich.edu/bitstream/2027.42/116722/1/antoun_1.pdf

Antoun, C., Conrad, F. G., Couper, M. P., \& West, B. T. (2019). Simultaneous estimation of multiple sources of error in a smartphone-based survey. Journal of Survey Statistics and Methodology, 7, 93-117.

Barnette, J. J. (1999). Nonattending respondent effects on internal consistency of selfadministered surveys: A Monte Carlo simulation study. Educational and Psychological Measurement, 59, 38-46.

Bowling, N. A., Huang, J. L., Bragg, C. B., Khazon, S., Liu, M., \& Blackmore, C. E. (2016). Who cares and who is careless? Insufficient effort responding as a reflection of respondent personality. Journal of Personality and Social Psychology, 111, 218-229.

Brosnan, K., Grün, B., \& Dolnicar, S. (2017). PC, Phone or Tablet?: Use, Preference and Completion Rates for Web Surveys. International Journal of Market Research, 59, 3555.

Buskirk, T. D., \& Andrus, C. (2014). Making mobile browser surveys smarter: Results from a randomized experiment comparing online surveys completed via computer or smartphone. Field Methods, 26, 322-342. 
Casler, K., Bickel, L., \& Hackett, E. (2013). Separate but equal? A comparison of participants and data gathered via Amazon's MTurk, social media, and face-to-face behavioral testing. Computers in Human Behavior, 29, 2156-2160.

Colquitt, J. A. (2001). On the dimensionality of organizational justice: a construct validation of a measure. Journal of Aapplied Psychology, 86, 386.

Couper, M. P., \& Peterson, G. J. (2017). Why do web surveys take longer on smartphones?. Social Science Computer Review, 35, 357-377.

Curran, P. G. (2016). Methods for the detection of carelessly invalid responses in survey data. Journal of Experimental Social Psychology, 66, 4-19.

De Beuckelaer, A., \& Lievens, F. (2009). Measurement equivalence of paper-and-pencil and Internet organisational surveys: A large scale examination in 16 countries. Applied Psychology, 58, 336-361.

de Bruijne, M. \& Wijnant, A. (2014) Mobile response in web panels. Social Science Computer Review, 32, 728-742.

de Bruijne, M., \& Wijnant, A. (2013). Comparing survey results obtained via mobile devices and computers: An experiment with a mobile web survey on a heterogeneous group of mobile devices versus a computer assisted web survey. Social Science Computer Review, 31, 482-504.

de Leeuw, E. \& de Heer, W. (2002) Trends in household survey nonresponse: a longitudinal and international perspective, in Groves, R.M., Dillman, D.A., Eltinge, J.L. \& Little, R.J.A. (eds) Survey Nonresponse (pp. 41-54). New York: Wiley.

DeSimone, J. A., Harms, P. D., \& DeSimone, A. J. (2015). Best practice recommendations for data screening. Journal of Organizational Behavior, 36, 171-181.

Dodou, D., \& de Winter, J. C. (2014). Social desirability is the same in offline, online, and paper surveys: A meta-analysis. Computers in Human Behavior, 36, 487-495.

Donnellan, M. B., Oswald, F. L., Baird, B. M., \& Lucas, R. E. (2006). The mini-IPIP scales: 
tiny-yet-effective measures of the Big Five factors of personality. Psychological assessment, 18, 192-203.

Fang, J., Wen, C., \& Prybutok, V. (2014). An assessment of equivalence between paper and social media surveys: The role of social desirability and satisficing. Computers in Human Behavior, 30, 335-343.

Francavilla, N. M., Meade, A. W., \& Young, A. L. (2019). Social Interaction and InternetBased Surveys: Examining the Effects of Virtual and In-Person Proctors on Careless Response. Applied Psychology, 68, 223-249.

Goldammer, P., Annen, H., Stöckli, P. L., \& Jonas, K. (in press). Careless responding in questionnaire measures: Detection, impact, and remedies. The Leadership Quarterly, https://doi.org/10.1016/j.leaqua.2020.101384.

Gollwitzer, M., Christ, O., \& Lemmer, G. (2014). Individual differences make a difference: On the use and the psychometric properties of difference scores in social psychology. European Journal of Social Psychology, 44, 673-682.

Grau, I., Ebbeler, C., \& Banse, R. (2019). Cultural Differences in Careless Responding. Journal of Cross-Cultural Psychology, 50, 336-357.

Groves, R.M. (2006) Nonresponse rates and nonresponse bias in household surveys. Public Opinion Quarterly, 70, 646-675.

Henrich, J., Heine, S. J., \& Norenzayan, A. (2010). The weirdest people in the world?. Behavioral and Brain Sciences, 33, 61-83.

Hoonakker, P., \& Carayon, P. (2009). Questionnaire Survey Nonresponse: A Comparison of Postal Mail and Internet Surveys. International Journal of Human-Computer Interaction, 25, 348-373.

Huang, J. L., Bowling, N. A., Liu, M., \& Li, Y. (2015). Detecting insufficient effort responding with an infrequency scale: Evaluating validity and participant reactions. Journal of Business and Psychology, 30, 299-311. 
Huang, J. L., Curran, P. G., Keeney, J., Poposki, E. M., \& DeShon, R. P. (2012). Detecting and deterring insufficient effort responding to surveys. Journal of Business and Psychology, 27, 99-114.

Huang, J. L., Liu, M., \& Bowling, N. A. (2014). Insufficient effort responding: Examining an insidious confound in survey data. Journal of Applied Psychology, 100, 828-845.

Johnson, J. A. (2005). Ascertaining the validity of individual protocols from web-based personality inventories. Journal of Research in Personality, 39, 103-129.

Lee, C., \& Coughlin, J. F. (2015, September). Generational differences in adoption and use of information and communications technology. In Proceedings of the Human Factors and Ergonomics Society Annual Meeting (Vol. 59, No. 1, pp. 892-896). Sage CA: Los Angeles, CA: SAGE Publications.

Lee, H., Kim, S., Couper, M. P., \& Woo, Y. (2019). Experimental comparison of PC Web, Smartphone Web, and Telephone Surveys in the new technology era. Social Science Computer Review, 37, 234-247.

Lehmann, R., Denissen, J. J., Allemand, M., \& Penke, L. (2013). Age and gender differences in motivational manifestations of the Big Five from age 16 to 60. Developmental Psychology, 49, 365-383.

Leung, K., Brew, F. P., Zhang, Z. X., \& Zhang, Y. (2010). Harmony and conflict: A crosscultural investigation in China and Australia. Journal of Cross-Cultural Psychology, 42, $795-816$.

Lugtig, P., \& Toepoel, V. (2015). The use of PCs, smartphones, and tablets in a probabilitybased panel survey effects on survey measurement error. Social Science Computer Review, 34, 78-94.

Maniaci, M. R., \& Rogge, R. D. (2014). Caring about carelessness: Participant inattention and its effects on research. Journal of Research in Personality, 48, 61-83.

Mavletova, A., \& Couper, M. P. (2013). Sensitive topics in PC web and mobile web surveys: 
is there a difference?. In Survey Research Methods, 7, 191-205.

Meade, A.W. \& Craig, S.B. (2012). Identifying careless responses in survey data. Psychological Methods, 17, 437- 455.

Morey, L. C., \& Hopwood, C. J. (2004). Efficiency of a Strategy for Detecting Back Random Responding on the Personality Assessment Inventory. Psychological Assessment, 16, $197-200$

Oetzel, J., Ting-Toomey, S., Masumoto, T., Yokochi, Y., Pan, X., Takai, J., \& Wilcox, R. (2001). Face and facework in conflict: A cross-cultural comparison of China, Germany, Japan, and the United States. Communication Monographs, 68, 235-258.

Olson, K. E., O'Brien, M. A., Rogers, W. A., \& Charness, N. (2011). Diffusion of Technology: Frequency of Use for Younger and Older Adults. Ageing International, 36, $123-145$.

Oppenheimer, D. M., Meyvis, T., \& Davidenko, N. (2009). Instructional manipulation checks: Detecting satisficing to increase statistical power. Journal of Experimental Social Psychology, 45, 867-872.

Paulhus, D. L. (1984). Two-component models of socially desirable responding. Journal of Personality and Social Psychology, 46, 598.

Peterson, G., Griffin, J., LaFrance, J., \& Li, J. (2017). Smartphone Participation in Web Surveys. In P.P. Biemer, E. Leeuw, S. Eckman, B. Edwards, F. Kreuter, L.E. Lyberg, N.C. Tucker and B.T. West (Eds.) Total survey error in practice (pp. 203-233). New Jersey: John Wiley \& Sons.

Prensky, M. (2001). Digital natives, digital immigrants part 1. On the horizon, 9, 1-6.

Sax, L. J., Gilmartin, S. K., \& Bryant, A. N. (2003). Assessing response rates and nonresponse bias in web and paper surveys. Research in Higher Education, 44, 409432.

Schmitt, M., Gollwitzer, M., Maes, J., \& Arbach, D. (2005). Justice Sensitivity. European 
Journal of Psychological Assessment, 21, 202-211

Stöber, J., Dette, D. E., \& Musch, J. (2002). Comparing continuous and dichotomous scoring of the Balanced Inventory of Desirable Responding. Journal of Personality Assessment, 78, 370-389.

Stocké, V. (2006). Attitudes toward surveys, attitude accessibility and the effect on respondents' susceptibility to nonresponse. Quality and Quantity, 40, 259-288.

Toepoel, V., \& Lugtig, P. (2014). What happens if you offer a mobile option to your web panel? Evidence from a probability-based panel of Internet users. Social Science Computer Review, 32, 544-560.

Tortora, R. (2009) Attrition in consumer panels, in Lynn, P. (Ed.) Methodology of Longitudinal Surveys (pp. 235-249). Chichester: John Wiley and Sons Ltd.

Tourangeau, R., Maitland, A., Rivero, G., Sun, H., Williams, D., \& Yan, T. (2017). Web surveys by smartphone and tablets: Effects on survey responses. Public Opinion Quarterly, 81, 896-929.

Ward, M. K., \& Meade, A. W. (2018). Applying social psychology to prevent careless responding during online surveys. Applied Psychology, 67, 231-263.

Ward, M. K., \& Pond III, S. B. (2015). Using virtual presence and survey instructions to minimize careless responding on Internet-based surveys. Computers in Human Behavior, 48, 554-568.

Ward, M. K., Meade, A. W., Allred, C. M., Pappalardo, G., \& Stoughton, J. W. (2017). Careless response and attrition as sources of bias in online survey assessments of personality traits and performance. Computers in Human Behavior, 76, 417-430.

Wells, T., Bailey, J. T., \& Link, M. W. (2013). Filling the void: Gaining a better understanding of tablet-based surveys. Survey Practice, 6, 1-9.

Wells, T., Bailey, J. T., \& Link, M. W. (2014). Comparison of smartphone and online computer survey administration. Social Science Computer Review, 32, 238-255. 
Woods, C. (2006). Careless responding to reverse-worded items: Implications for confirmatory factor analysis. Journal of Psychopathology and Behavioral Assessment, $28,189-194$.

Yun, G. W., \& Trumbo, C. W. (2000). Comparative response to a survey executed by post, email, \& web form. Journal of Computer-mediated Communication, 6, 0-0.

Zhang, C., \& Conrad, F. (2014). Speeding in web surveys: The tendency to answer very fast and its association with straightlining. In Survey Research Methods, 8, 127-135 


\section{Appendix A}

\section{Self-Reported Effort scale}

1. I worked to the best of my abilities in this study.

2. I put forth my best effort in responding to this survey.

3. I would be interested in reading about the results of this study.

4. I'm in a hurry right now. (R) 


\section{Figure Captions}

Figure 1. Differences between mode in careless responding for the Chinese working adults sample.

Figure 2. Differences between mode in careless responding for the German student sample.

Figure 3. Differences between mode in careless responding for the Chinese speaking student sample. 


\section{Tables}

\section{Table 1}

Sample information for the four sampling locations.

\begin{tabular}{lcccccc} 
Age & N & Minimum & Maximum & Mean & $\begin{array}{c}\text { Std. } \\
\text { Deviation }\end{array}$ & \%female \\
\hline Chinese working & 78 & 21 & 56 & 36,03 & 9,78 & 57,7 \\
German students & 117 & 18 & 49 & 21,07 & 4,64 & 88,8 \\
$\begin{array}{l}\text { Chinese students, } \\
\text { University 1 }\end{array}$ & 74 & 19 & 22 & 20,23 & 0,79 & 90,5 \\
$\begin{array}{l}\text { Chinese students, } \\
\text { University 2 }\end{array}$ & 39 & 20 & 24 & 21,13 & 1,08 & 25,6 \\
\hline
\end{tabular}




\section{Table 2}

\section{Table 2a}

Correlations Among Variables for Chinese Working Adults Sample

\begin{tabular}{llllllllll}
\hline Variable & $\mathrm{N}$ & 1 & 2 & 3 & 4 & 5 & 6 & 7 & 8 \\
\hline 1. Long-string Mean & 78 & 1 & & & & & & & \\
2. Mahalanobis distance & 78 & $-0,088$ & 1 & & & & & & \\
3. Psychometric synonyms & 78 & 0,118 &,$- 235^{*}$ & 1 & & & & & \\
4. Psychometric antonyms & 76 & 0,134 & $-0,063$ & $-0,207$ & 1 & & & & \\
5. Person total correlation & $\mathrm{a}$ & 78 & $-0,190$ &,$- 225^{*}$ &, $397^{* *}$ &,$- 520^{* *}$ & 1 & & \\
6. Self reported effort & 78 & $-0,168$ &, $280^{*}$ & 0,104 & $-0,142$ & 0,036 & 1 & & \\
7. Instructed Response Items & 78 & 0,145 & $-0,058$ &, $258^{*}$ & 0,061 & $-0,119$ & $-0,105$ & 1 & \\
8. Use me? Y/N & 78 & 0,059 & 0,214 & 0,103 & 0,101 & $-0,096$ &, $320^{* *}$ & 0,031 & 1 \\
Careless Responding Index & 78 &, $421^{* *}$ &, $291^{* *}$ &,$- 459^{* *}$ &, $578^{* *}$ &,$- 727^{* *}$ &,$- 418^{* *}$ &, $385^{* *}$ & 0,012 \\
\hline
\end{tabular}

** Correlation is significant at the 0.01 level (2-tailed). * Correlation is significant at the 0.05 level (2-tailed).

1-7 included in the CR index, ${ }^{a}$ Reverse coded in the calculation of CR index

\section{Table 2b}

Correlations Among Variables for German students

\begin{tabular}{|c|c|c|c|c|c|c|c|c|c|}
\hline Variable & & 1 & 2 & 3 & 4 & 5 & 6 & 7 & 8 \\
\hline 1. Long-string Mean & 117 & 1 & & & & & & & \\
\hline 2. Mahalanobis distance & 117 & 0,012 & 1 & & & & & & \\
\hline 3. Psychometric synonyms ${ }^{\mathrm{a}}$ & 117 & $215^{*}$ & 0,029 & 1 & & & & & \\
\hline 4. Psychometric antonyms & 115 & $-0,018$ & 0,085 & 0,052 & 1 & & & & \\
\hline 5. Person total correlation ${ }^{a}$ & 117 & $-0,043$ &,$- 554 * *$ &,$- 221 *$ &,$- 355^{* *}$ & 1 & & & \\
\hline 6. Self reported effort ${ }^{\mathrm{a}}$ & 116 & 0,157 & 0,142 & 0,117 & $-0,166$ & $-0,104$ & 1 & & \\
\hline 7. Instructed Response Items & 117 & 0,029 & 0,074 & $-0,022$ & $-0,024$ & $-0,069$ &,$- 209 *$ & 1 & \\
\hline 8. Use me? Y/N & 117 & $-0,067$ & $-0,013$ & 0,021 & 0,019 & $-0,005$ &,$- 210 *$ & 0,146 & 1 \\
\hline Careless Responding Index & 117 & ,199* &, $517 * *$ &,$- 245^{* *}$ &, $493 * *$ &,$- 528^{* *}$ &,$- 383^{* *}$ &, $495 * *$ & 0,088 \\
\hline
\end{tabular}

** Correlation is significant at the 0.01 level (2-tailed). * Correlation is significant at the 0.05 level (2-tailed).

1-7 included in the CR index, ${ }^{\text {a }}$ Reverse coded in the calculation of CR index 


\section{Table 2c}

Correlations Among Variables for Chinese speaking students

\begin{tabular}{|c|c|c|c|c|c|c|c|c|c|}
\hline Variable & & 1 & 2 & 3 & 4 & 5 & 6 & 7 & 8 \\
\hline 1. Long-string Mean & 113 & 1 & & & & & & & \\
\hline 2. Mahalanobis distance & 113 &,$- 422 * *$ & 1 & & & & & & \\
\hline 3. Psychometric synonyms ${ }^{a}$ & 108 & $-0,033$ & 0,05 & 1 & & & & & \\
\hline 4. Psychometric antonyms & 113 &, $247 * *$ & $-0,108$ & 0,029 & 1 & & & & \\
\hline 5. Person total correlation ${ }^{\mathrm{a}}$ & 113 & $-0,061$ & $-0,171$ & 0,111 &,$- 225^{*}$ & 1 & & & \\
\hline 6. Self reported effort ${ }^{a}$ & 113 &,$- 300 * *$ &, $288^{* *}$ & 0,02 &,$- 370 * *$ &, $293 * *$ & 1 & & \\
\hline 7. Instructed Response Items & 113 &, $456 * *$ & $-0,11$ & $-0,052$ &, $233^{*}$ &,$- 208^{*}$ &,$- 215^{*}$ & 1 & \\
\hline 8. Use me? Y/N & 113 &, $196^{*}$ & $-0,013$ & $-0,077$ &, $222 *$ & $-0,091$ &,$- 287 * *$ & 0,129 & 1 \\
\hline Careless Responding Index & 113 &, $516^{* *}$ & 0,036 &,$- 367 * *$ &, $609 * *$ &,$- 615^{* *}$ &,$- 587 * *$ &, $626 * *$ &, $308 * *$ \\
\hline
\end{tabular}

** Correlation is significant at the 0.01 level (2-tailed). * Correlation is significant at the 0.05 level (2-tailed).

1-7 included in the CR index, ${ }^{a}$ Reverse coded in the calculation of CR index 
Table 3

Discriptive Statistics indicies, in three samples

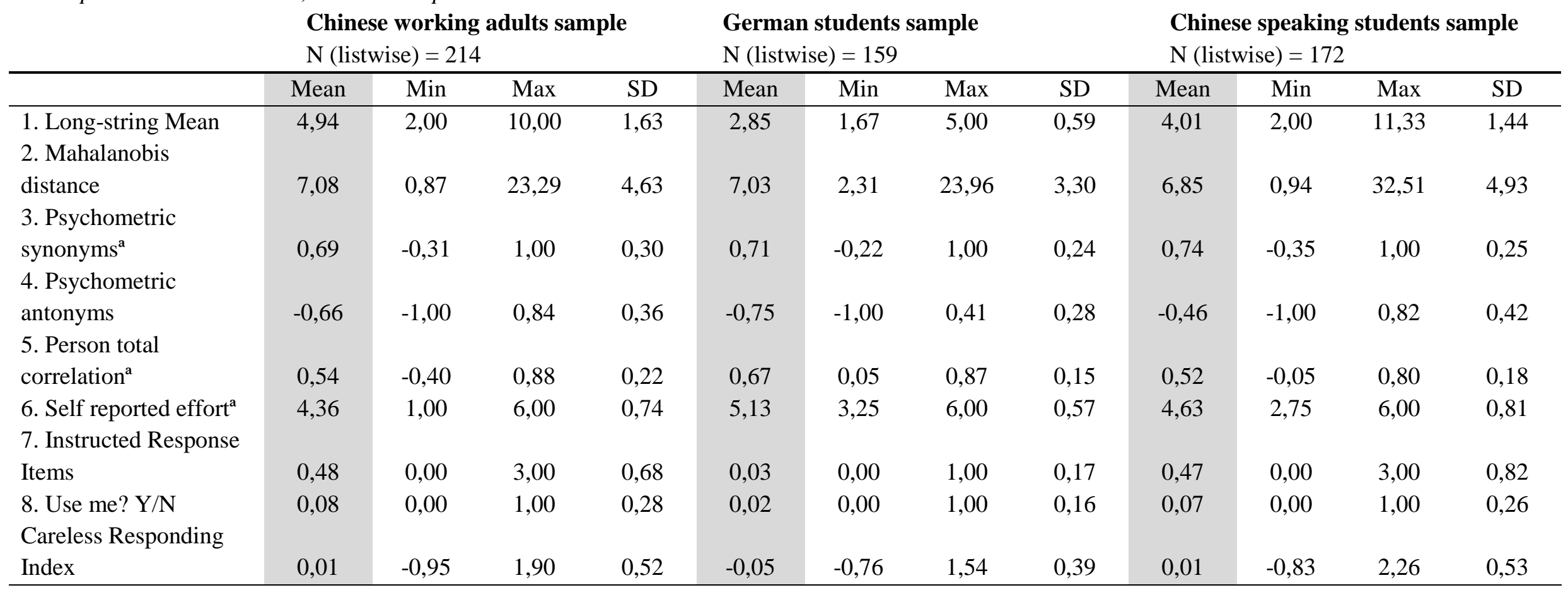

1-7 included in the CR index, ${ }^{a}$ Reverse coded in the calculation of CR index 